\title{
Dorsal Hand Vein Extraction in Uncontrolled Environment
}

\author{
Nisha Charaya ${ }^{1}$, Anil Kumar $^{2}$, Priti Singh ${ }^{3}$ \\ Department of ECE \\ Amity University, Gurgaon \\ Haryana, India
}

\begin{abstract}
Biometrics is an inseparable part of our day to day life. A major development in this area has been observed in past few decades. Over the recent years, dorsal hand veins have emerged as a promising biometric attribute due to its universality, stability and anti-forgery characteristics. However, detecting the veins of different thickness under different illumination is a challenging task. The traditional vein extraction approaches based on thresholding does not find their applicability in these situations. This paper presents a hybrid approach for vein segmentation for these hand images. The proposed approach is a combination of two techniques, i.e. repeated line tracking and maximum curvature points. The technique has been tested over Bosphorus hand vein dataset which contains 1575 images of different age groups captured under different illumination conditions. From the results, it is evident that this technique is suitable to extract vein pattern from all types of images. Further, these images have yielded an accuracy of more than $\mathbf{9 8 \%}$ when subjected to feature extraction and classification steps.
\end{abstract}

Keywords-Biometrics; security; forgery; hand vein; vein segmentation; vein extraction; thick veins; unclear veins

\section{INTRODUCTION}

With the advances in technology and implementation of automation everywhere, security has been put at stake and needs to be ensured before bringing a new technology into practice. Starting from minor to major, all the activities in our lives has become automated with the help of biometrics. Attendance marking systems in offices, online transactions in banks and door opening at home; everything has become fast and automatic. But this automation requires a perfect, accurate and precise method to identify a person[1][2]. The technique to identify or verify a person with the help of physiological or behavioral attributes is known as biometrics. Iris, veins, fingerprints, and face are examples of physiological biometrics whereas voice, gait and DNA are examples of behavioral biometrics. A biometric system is user-convenient as compared to the traditional methods as it doesn't require be kept with safety or crammed all the times [3].

Hand-veins are seeking researcher's attention due to its attracting characteristics of user convenience and anti-forgery. Under the human skin, there is vein pattern which consists of various networks of blood vessels. The structure of vascular pattern in human body parts is diverse and stable over time [4]. Further it is present beneath skin which cannot be visible directly with bare eyes of humans. Related to other biometric features, vein pattern are very difficult to recognize. Still its characteristics like uniqueness, contactless, universality and anti-forgery are strong enough to hide the challenges in its use. One of the main challenges in vein pattern based biometric system is to acquire images of vein pattern quickly without involving harmful and expensive devices which has been resolved by the near infra-red cameras.

Another major challenge is extracting the vein pattern from the images captured under poor illumination, with different hand postures and for people of different age group [5]. The thickness of hand veins vary with age and it gets affected due to some common diseases like diabetes and hypertension. In addition to these, irregular shading, optical blurring and skin scattering are some unavoidable factors which deteriorates the vein recognition and overall accuracy of the system. Though a lot of significant work has been contributed by researchers, yet an effective technique which can extract vein pattern from all types of images needs to be devised. This raises the need for finding a vein detection technique which is capable of extracting veins of varying thickness from the unclear images acquired from hands kept in different postures.

In this paper, a hybrid approach for the same is presented which is a merger of two techniques i.e. repeated line tracking method and maximum curvature point's method. The repeated line tracking method is based on tracing the dark lines by examining the cross section profile. It has proved to be effective for unclear images but faces issues for thick/thin veins. On the other hand, maximum curvature point method detects the vein based on the points having maximum curvature. This method is quite satisfactory for detecting the veins of different thickness but fails when the images are unclear. As the former one is suitable for unclear images while the latter is for thick/thin veins, so a combination of these two is proposed which has proved to be efficient for detecting veins from all types of hand images.

The main objective of this paper is to devise a vein extraction technique which can detect the veins of varying thickness from unclear images acquired in uncontrolled environment.

The rest of this paper is organized as follows. Section II discusses related work on this topic. The general methodology is described in Section III. Section IV depicts the proposed method and Section V contains simulation results. Finally, Section VI concludes the paper. 


\section{LITERATURE STUDY}

The generalized and most basic way to detect vein pattern is by identifying the dark lines. It is done by thresholding method. A suitable threshold is selected and all the pixel values are compared with the threshold. Based on the comparison, it is categorized as background or vein. The most common thresholding method is OTSU's thresholding which has been adopted by many researchers to extract vein pattern [6]. However, the traditional method fails for images captured in uncontrolled environments in different hand postures.

Apart from thresholding, some different concept has also been devised to detect the vein patterns. The repeated line tracking method has been implemented by Miura (2004) to extract the finger veins which was able to extract veins in the presence of irregular shading [7], [8].

In 2007, Zhao applied local dynamic threshold method for vein segmentation which was based on extracting the veins by calculating mean and variance of the points in the neighborhood [9].

Zhong, Shao and Liu (2018) adopted the curvature point algorithm to extract the veins in an uncontrolled environment which was earlier deployed by Miura(2005) to extract finger vein pattern[10][11]]. This algorithm is based on the concept that, in each grey profile of the grey image the distribution of grayscale values is a concave curve and the curvature value of point on the vein is greater than zero. So, the veins can be extracted by finding the pixels whose curvature values are greater than zero. The curvature point used deeper data features, which largely eliminated the effects of noise and retained more venous details [4].

Li (2010) used a local dynamic thresholding named NiBlack to segment the vein image[12]. To overcome the effect of uneven surfaces which leads to dark regions in DHV image, Shang-Jen Chuang (2017) applied global-local threshold algorithm [13].

BELEAN (2017) applied Hough transform (VP-HT) to determine the line segments in hand vein image. This technique is based on a global description of image features [14].

The majority of the methods for DHV recognition are devised on the basis of geometrical strategies and thresholding. However, optical blurring, hand vein postures and skin scattering are the major issues which may result into irregular shadings that may degrade the accuracy of recognition. A comparative analysis of existing techniques is depicted in Table 1 which clearly shows the lack of a technique that can extract vein pattern from thick/thin veins and unclear images acquired in uncontrolled environment.

All the techniques have their own significances but none of them can be used globally to extract vein pattern from all type of hand vein images due to their own applicability to particular images.
TABLE I. COMPARATIVE ANALYSIS OF EXISTING VEIN SEgMENTATION TECHNIQUES

\begin{tabular}{|l|l|l|l|}
\hline Year & Authors & Technique Used & Remarks \\
\hline 2004 & $\begin{array}{l}\text { Miura, Nagasaka, } \\
\text { and Miyatake }\end{array}$ & $\begin{array}{l}\text { Repeated line } \\
\text { tracking }\end{array}$ & $\begin{array}{l}\text { Tested on finger veins, } \\
\text { not suitable for thick } \\
\text { veins }\end{array}$ \\
\hline 2007 & Zhao et. al & $\begin{array}{l}\text { Local dynamic } \\
\text { thresholding }\end{array}$ & $\begin{array}{l}\text { Not suitable for } \\
\text { uncontrolled } \\
\text { environment }\end{array}$ \\
\hline 2012 & $\begin{array}{l}\text { Li, Wang, and } \\
\text { Jiang }\end{array}$ & $\begin{array}{l}\text { NiBlack } \\
\text { thresholding } \\
\text { OTSU }\end{array}$ & $\begin{array}{l}\text { Not suitable for noisy } \\
\text { images acquired in } \\
\text { uncontrolled } \\
\text { environment }\end{array}$ \\
\hline 2017 & $\begin{array}{l}\text { Shang-Jen } \\
\text { Chuang }\end{array}$ & $\begin{array}{l}\text { Not suitable for noisy } \\
\text { images acquired in } \\
\text { uncontrolled } \\
\text { environment }\end{array}$ \\
\hline 2017 & Bellean et al. & Hough transform & $\begin{array}{l}\text { Not suitable for noisy } \\
\text { images acquired in } \\
\text { uncontrolled } \\
\text { environment }\end{array}$ \\
\hline 2018 & $\begin{array}{l}\text { Zhot tested for noisy } \\
\text { and thick veins images }\end{array}$ \\
\hline
\end{tabular}

Extraction of vein pattern is an essential requirement for designing a hand vein based human authentication system. So, a poorly extracted pattern cannot result into an accurate biometric system. This raises the need for a global method of vein detection which can be used for all kind of hand images that are acquired with different postures, ages and illumination.

To achieve this, a new vein detection technique is proposed in this paper.

\section{GENERAL METHODOLOGY}

A general biometric system is comprised of several components as shown in fig 1 . The sensor element collects the unprocessed biometric data from an individual that may be in the form of an image, video, audio or some other signal. This unprocessed data is passed through the pre-processing block where it is refined, improved and made ready to be processed and utilized [15]. The feature extraction block gathers a unique set of features to represent the signal. The extracted feature set is labeled with user identity and stored in the database during user enrolment phase. The matching block is responsible for performing comparison between the data presented for identification and the stored data. As an outcome of comparison, a matching score is generated. The decision block categorizes the presented data as identified or unidentified based on its matching score value.

Image pre-processing specifically vein extraction plays an important role in the overall system performance. The images captured in uncontrolled environment contain noise due to variation in illumination, optical blurring and bone shadows [4]. Also, it is challenging to extract veins from all types of images with different hand postures and varying vein thickness. So, it becomes mandatory to pre-process the images. 


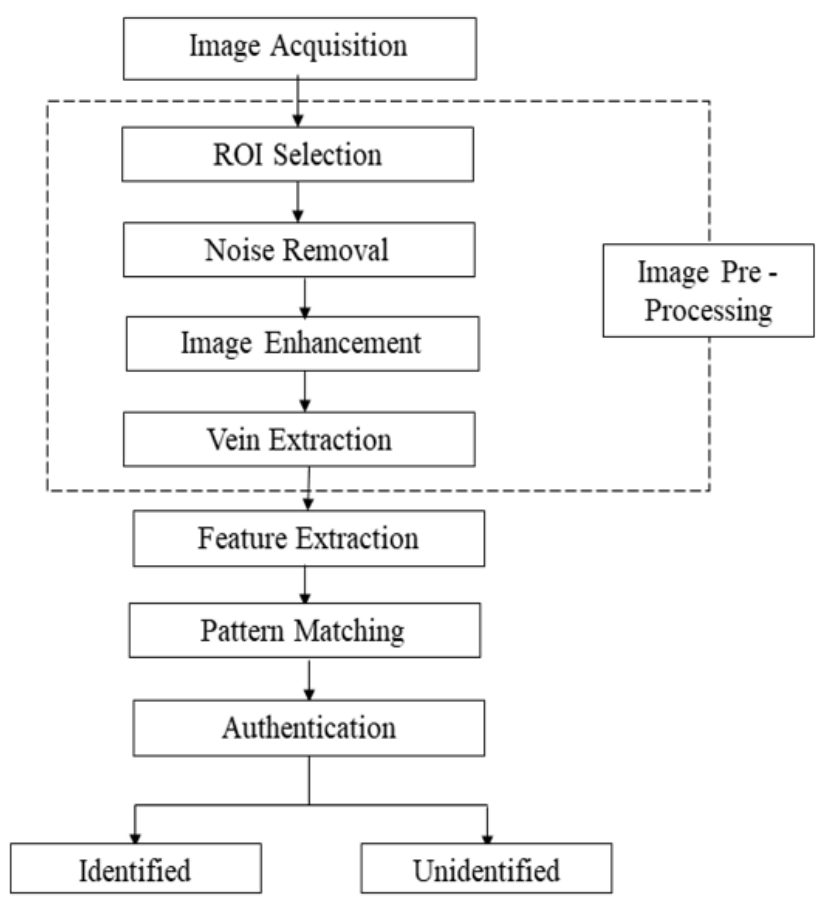

Fig. 1. Flow Chart of Hand vein based Authentication System.

For this, the images acquired by near infra-red camera are first converted to gray scale images. After this, histogram equalization is performed to make the image uniformly illuminated. Noise is removed by the application of median filter and the images are enhanced by morphological 'open' operations. Once enhanced hand image has been obtained, it is used to extract the region of interest (ROI) for further processing.

The ROI is a small area selected and extracted out of whole image for further processing. The size of image and the number of images in a database is very large which makes it time consuming to process the complete data as well as its memory requirements are larger. For these reasons, a particular small region is selected out of the whole image from which the features are extracted and stored for database creation and matching purpose. Generally, the ROI is selected around the centre of image so that it is present in all the images and is not affected by the position and placement of hand. In this work, ROI is extracted from the segmented hand image and is chosen such that the most affected and corrupted area is removed from the image and all the important information is retained. It comes out to be a rectangular or square region which is equidistant from all the margins in all directions. Once the region of interest is obtained, the proposed method is employed to extract the vein pattern from the selected regions.

\section{Proposed Method}

Vein segmentation is an important and essential step in hand vein based authentication system as the system performance is directly dependent on it. The proposed technique of vein detection is a combination of two techniques i.e. repeated line tracking method and maximum curvature point method. The dorsal hand images are passed through them one by one and the resultant images are obtained. Finally the resultant images obtained by the two methods are overlaid upon each other to get the vein pattern extracted.

\section{A. Repeated Line Tracking Method}

The repeated tracking of dark lines is performed to facilitate extraction of hand-vein patterns using non-uniform images. The hand veins are extracted from the non uniform images based on tracking the veins in all directions starting from a seed pixel. The line-tracking operation is initiated from any pixel in the captured image. The current position of a tracking pixel is called as the "current tracking point", and it is moved along the dark line pixel by pixel. The vein appears as a valley in its cross sectional profile as shown in fig 2 . The depth of valley is affected by the shading in the image but still it can be detected. It makes this method a robust method that can be used for extracting veins from non-uniform images. The depth of the cross-sectional profile around the current tracking point is checked to decide whether this point lies on a dark line or not. If this cross sectional profile resembles a valley, the tracking point is on a dark line. Further, the cross section profile is observed for different angles and the one with deepest profile is retained to provide the direction of tracking. After this, the current tracking point is updated with the closest pixel in that direction. If a valley is detected again at the updated tracking point, the tracking is continued in the direction of deepest valley and this result in extraction of a vein or part of a vein. Else if valley is not detected in any direction, this means that the updated pixel is not on a dark line and a new tracking operation is started at some other position. This tracking process is depicted in fig 3 showing current tracking point, direction and the cross section profile. A single line tracking operation will yield only a part of veins, so to gather and recognize all the hand veins, tracking is performed at various random starting positions across the whole image.

Sometimes, a tracking operation may also track a region of noise but there are more chances of tracking a dark line which represents a vein. A matrix named the "locus space" is created to store the number of times that each pixel has become the current tracking point. The size of the locus space is determined by the number of pixels in the captured images. Matrix elements are obtained by recording the total count in which the pixel has become the current tracking point against each pixel. Therefore, a frequently tracked element of the locus space has a higher value. The hand veins are obtained as chains of these high value positions in the locus space.
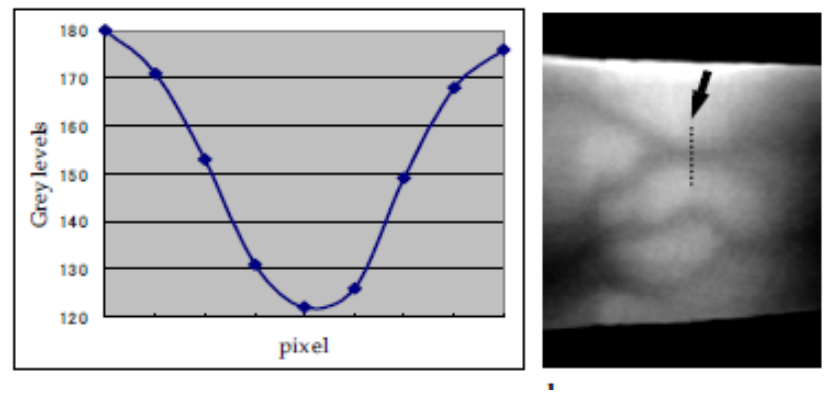

Fig. 2. Cross-sectional Profile and Pixel Position [9]. 


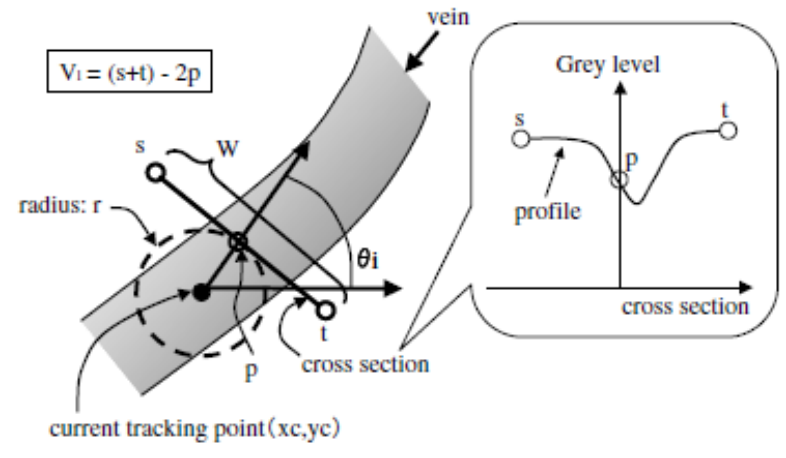

Fig. 3. Detection of Dark Line by Repeated Line Tracking [9].

The steps considered in recognizing the hand veins using repeated line tracking strategy is portrayed below:

Step 1: Discovering the starting point to move-direction attributes and start line tracking.

Step 2: Recognition of tracking direction by examining cross-sectional profile and updating tracking position along the dark line.

Step 3: Update count against a pixel in locus space whenever a pixel gets traced.

Step 4: Repeat step 1 to step 3.

Step 5: Acquiring the vein pattern via locus space.

This method has the advantage of quick processing and minimized computational time while extracting the vein pattern from unclear dorsal hand images with non-uniform illumination.

\section{B. Maximum Curvature Point Method}

The width of hand veins varies with several conditions like temperature, blood in the veins, physical conditions etc. Also with ageing, the vein thickness increases which produces difficult in tracking. To combat these issues, the maximum curvature points technique is employed to inspect the curvature of image and highlight center-lines of veins. The center-lines are determined by probing the position wherein the curvatures of a cross-sectional profile of a vein are locally maximal. These points are connected with each other to obtain the vein pattern.

The algorithm is processed with three steps which involve:

- Mining of centre positions of veins.

- Connecting centre positions.

- Image labelling.

The algorithm checks the cross-sectional profile of a hand vein image. As the vein is darker than its surroundings, the cross-sectional profile of a vein appears like a dent. It appears as a concave surface with large curvature. The curvature is large even for a thin vein. Therefore, even the thin veins get recognized by obtaining local maximum curvature points. Each local maximum position is assigned with a scare which is larger for a deeper profile.
The curvature $\mathrm{C}(\mathrm{z})$ of a cross sectional profile $\mathrm{P}_{\mathrm{H}}(\mathrm{z})$ can be represented as:

$\mathrm{C}(\mathrm{z})=\frac{\mathrm{d}^{2} \mathrm{P}_{\mathrm{H}}(\mathrm{z}) / \mathrm{dz}^{2}}{\left\{1+\left(\frac{\mathrm{dP}_{\mathrm{H}}(\mathrm{z})}{\mathrm{dz}}\right)^{2}\right\}^{3 / 2}}$

Where, $P_{H}(z)$ is obtained from the intensity of a pixel, $\mathrm{H}(\mathrm{x}, \mathrm{y})$ in any direction and $\mathrm{z}$ represents a direction in profile. $\mathrm{P}_{\mathrm{H}}(\mathrm{z})$ is related to $\mathrm{H}(\mathrm{x}, \mathrm{y})$ by a mapping function $\mathrm{T}_{\mathrm{rs}}$ [10].

The sign of $\mathrm{C}(\mathrm{z})$ is observed to determine the nature of curvature. The curvature is concave for a positive sign and convex for a negative sign of $\mathrm{C}(\mathrm{z})$. The points, where local maxima are obtained for a concave area, give the centre positions of the veins. All such points are stored as $\mathrm{z}_{\mathrm{k}}^{\prime}$ and a score is assigned to them. The assigned scores determine the probability that these points are on the veins. The score for any centre position is calculated as:

$\mathrm{S}\left(\mathrm{z}_{\mathrm{k}}^{\prime}\right)=\mathrm{C}\left(\mathrm{z}_{\mathrm{k}}^{\prime}\right) \times \mathrm{W}_{\mathrm{r}}(\mathrm{k})$

Where, $W_{r}(k)$ represents the width of concave region containing $\mathrm{z}_{\mathrm{k}}^{\prime}[10]$.

The centre positions of veins are joined together based on score values to obtain veins from group of points. The profiles are analyzed in all four directions to acquire the vein pattern. The vein pattern so obtained is binarized by thresholding method.

The hand veins recognized by the two methods are overlaid upon the original image and stored in the vector form represented as J. Each hand vein region is then subjected to feature extraction and classification for mining significant features and matching process respectively.

\section{Simulation Results}

The proposed technique has been implemented in MATLAB and tested over Bosphorus Hand database.. The database comprises of 1575 images including both left and right hand images captured under different postures and 219 are captured with a time drop of several months.

The database comprises 642 subjects having 6 images for a person, which contains three right-hand images and three lefthand images. The images are of different illumination condition and different postures.

Moreover, 276 subjects with three left-hand images only. Amongst total 918 subjects, 160 poses hand images with time drop of several months.

The proposed method has been tested for vein detection in normal hand images, images with thick veins, rotated hand images and translated hand images. The simulation results for the same are shown in figure 4-6.

The resultant images obtained from maximum curvature point method shown in fig 4, contains dark patches due to irregular shading. While repeated line tracking fails to extract vein pattern from thick vein images as shown in fig 5 . From the simulation results shown in fig 6 , it is observed that the proposed method can successfully detect vein pattern from all kind of images which can be further used for feature 
extraction and classification steps to develop the complete authentication system.

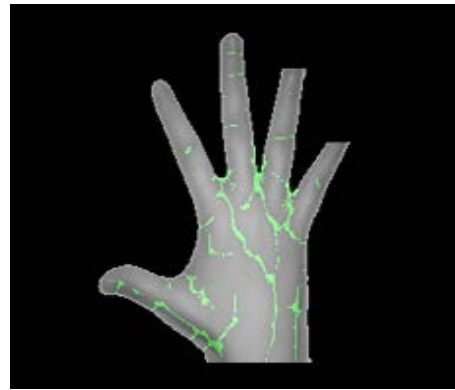

(a)

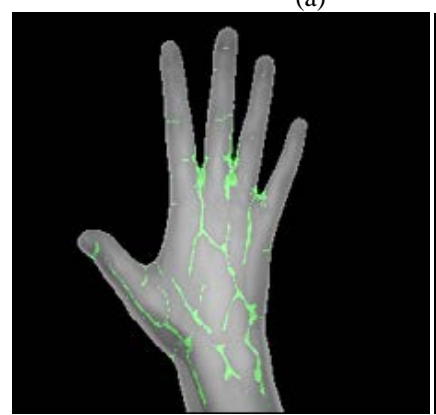

(c)

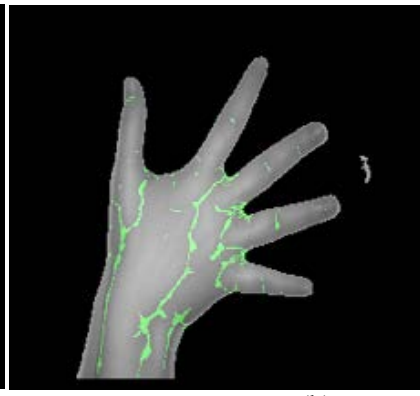

(b)

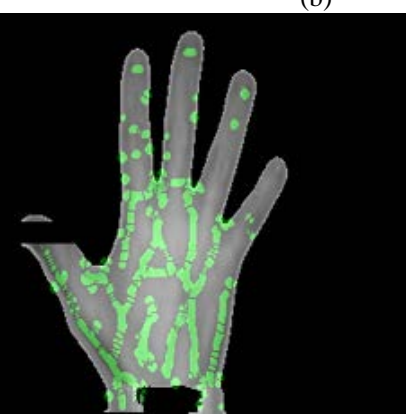

(d)
Fig. 4. Veins Extracted by Maximum Curvature Points Method for (a) Normal Image (b) Rotated Image (c) Translated Image (d) Thick Vein Image.

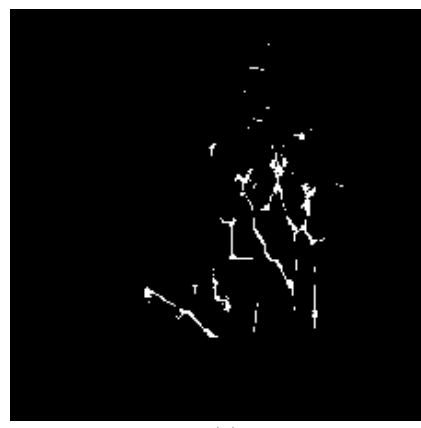

(a)

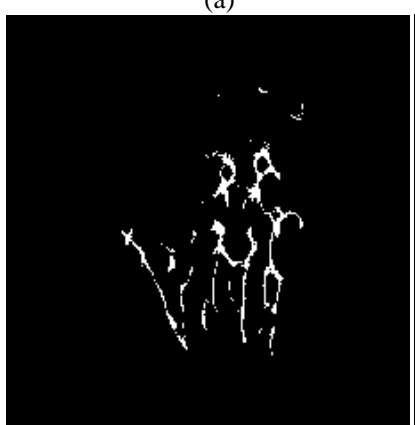

(c)

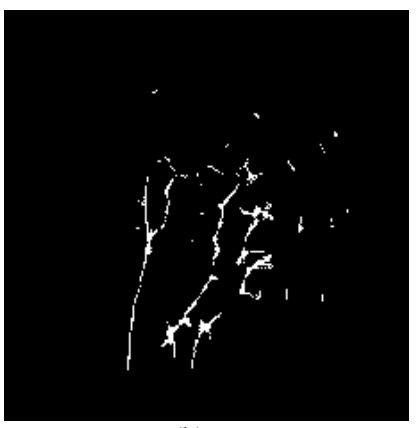

(b)

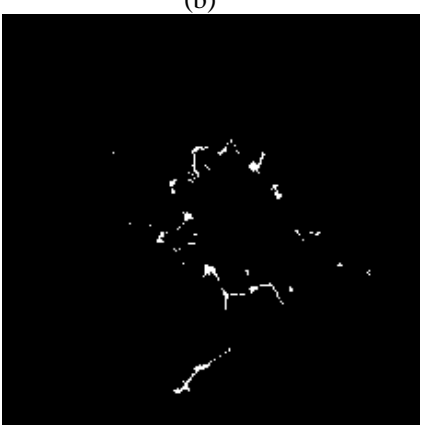

(d)
Fig. 5. Veins Extracted by Repeated Line Tracking Method for (a) Normal Image (b) Rotated Image (c) Translated Image (d) Thick Vein Image.

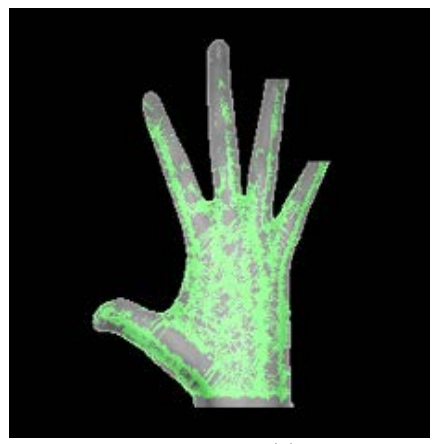

(a)

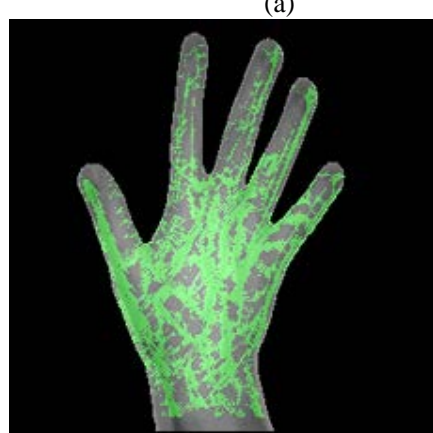

(c)

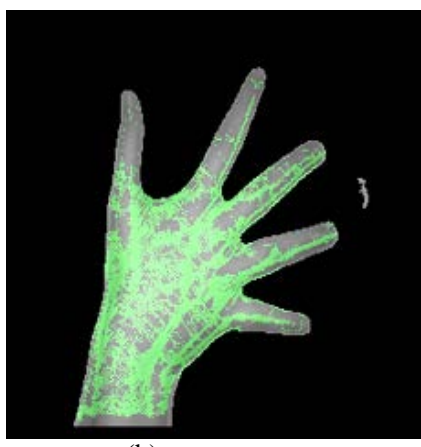

(b)

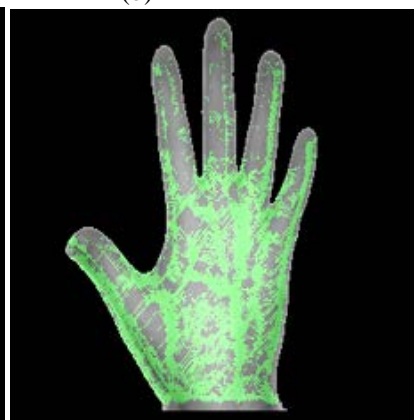

(d)
Fig. 6. Veins Extracted by Proposed Method for (a) Normal Image (b) Rotated Image (c) Translated Image (d) Thick Vein Image.

\section{CONCLUSION}

It is evident from the results that the proposed method can efficiently detect the vein pattern from thick veins and unclear images acquired in uncontrolled environment. This method can be used as a global method of vein segmentation for all kind of images. The vein pattern so produced when further tested for matching purpose by using convolutional neural network (CNN) for feature extraction and deepCNN for classification yields overall accuracy of above $98 \%$ for all type of images. However, this approach needs to be validated over practical noisy images and can be extended to the design of dorsal hand vein based biometric system.

\section{REFERENCES}

[1] K. Dharavath, F. A. Talukdar, and R. H. Laskar, "Study on biometric authentication systems, challenges and future trends: A review," 2013 IEEE Int. Conf. Comput. Intell. Comput. Res. IEEE ICCIC 2013, 2013.

[2] N. Charaya, "Human Authentication Based On Dorsal Hand Veins : A Review,” vol. 119, no. 16, pp. 2175-2185, 2018.

[3] N.Charaya, "Biometric Systems and Attributes: A review", Advancements \& Modern Innovations in Engineering and Technology (AMIET 2020), pp. 250-253.

[4] D. Zhong, H. Shao, and S. Liu, "Towards application of dorsal hand vein recognition under uncontrolled environment based on biometric graph matching,” IET Biometrics, vol. 8, no. 2, pp. 159-167, 2019.

[5] L. Wang and G. Leedham, "Near- and far- infrared imaging for vein pattern biometrics,” Proc. - IEEE Int. Conf. Video Signal Based Surveill. 2006, AVSS 2006, pp. 4-9, 2006. 
[6] H. Li, Y. Wang, and X. Jiang, "Dorsal hand vein recognition method based on multi-bit planes optimization,” vol. 1, Springer International Publishing, pp. 3-10, 2018.

[7] N. Miura, A. Nagasaka, and T. Miyatake, "Feature extraction of fingervein patterns based on repeated line tracking and its application to personal identification,” Mach. Vis. Appl., vol. 15, no. 4, pp. 194-203, Oct. 2004.

[8] N. Miura, A. Nagasaka, and T. Miyatake, "Feature extraction of finger vein patterns based on iterative line tracking and its application to personal identification,” Syst. Comput. Japan, vol. 35, no. 7, pp. 61-71, 2004.

[9] S. Zhao, Y. Wang, and Y. Wang, "Extracting hand vein patterns from low-quality images: A new biometric technique using low-cost devices," Proc. 4th Int. Conf. Image Graph. ICIG 2007, pp. 667-671, 2007.

[10] N. Miura, A. Nagasaka, and T. Miyatake, "Extraction of finger-vein patterns using maximum curvature points in image profiles," IEICE Trans. Inf. Syst., vol. E90-D, no. 8, pp. 1185-1194, 2007.
[11] "Miura et al. vein extraction methods-MATLAB Answers-MATLAB Central."[Online].Available: https://in.mathworks.com/matlabcentral/ fileexchange/35716-miura-et-al-vein-extraction-methods.[Accessed: 30Jan-2021].

[12] X. Li, X. Liu, and Z. Liu, "A dorsal hand vein pattern recognition algorithm,” Proc. - 2010 3rd Int. Congr. Image Signal Process. CISP 2010, vol. 4, no. 2, pp. 1723-1726, 2010.

[13] S. J. Chuang, "Vein recognition based on minutiae features in the dorsal venous network of the hand," Signal, Image Video Process., vol. 12, no. 3, pp. 573-581, 2018.

[14] B. Belean, M. Streza, S. Crisan, and S. Emerich, "Dorsal hand vein pattern analysis and Neural Networks for biometric authentication," Stud. Informatics Control, vol. 26, no. 3, pp. 305-314, 2017.

[15] A. K. Jain, P. Flynn, and A. A. Ross, "Handbook of Biometrics Handbook of Biometrics,” 2007. 